\title{
Synthesis of free-metal composites g-CN/GQDs with enhanced photocatalytic degradation of tetracycline
}

\author{
Kai Zheng ${ }^{1}$, Jianan $\mathrm{Chen}^{1}$, Xue $\mathrm{Gao}^{1}$, Xiaoqing $\mathrm{Cao}^{1}$, Shan $\mathrm{Wu}^{1}$, and Jixin $\mathrm{Su}^{1 *}$ \\ ${ }^{1}$ School of Environmental Science and Engineering, Shandong University, China
}

\begin{abstract}
Developing inexpensive and environmentally friendly no-metal photocatalyst is a promising strategy to addressing environmental issues. In this work, the metal-free catalyst g-CN/GQDs was synthesized through one-pot co-sintering method. The as-prepared photocatalysts showed enhanced photocatalytic activity for tetracycline (TC) degradation. Especially, the g-CN/GQDs-5 exhibited a nearly 30\% improvement in TC degradation within 60 min of visible light irradiation compared to g-CN, and the kinetic constant was 1.8 times that of $\mathrm{g}-\mathrm{CN}$. The enhanced performance was attributed to the introduction of GQDs enhance the visible light absorption ability and suppress the recombination of photo-generated charges of g-CN. The capture experiment of active species showed that superoxide radicals played a major role in the degradation of TC in this system.
\end{abstract}

\section{Introduction}

Antibiotics, as an antibacterial medicine, have been widely applied to control infection diseases in humans and animals[1]. Particularly, tetracycline (TC), a class of broad-spectrum antibiotics with a parent nucleus of pentane, has attracted great attention in the last few decades owing to its wide range of application, hardly mineralization, and potential threats to water ecosystem and terrestrial[2-5]. Unfortunately, TC cannot be effectively removed by traditional treatment technology due to its chemical stability, good solubility, and nonbiodegradability. Thus, it is meaningful to explore a proper way to sweep them. Semiconductor photocatalysis has been deemed as a possible and promising method to solve universal environmental predicaments, owing to the mild reaction conditions, low cost, and the stability of physicochemical properties[6-8].

Graphitic carbon nitride ( $\mathrm{g}-\mathrm{CN})$, a metal-free visible responsive polymer photomaterial, has been widely studied in the removal of various contaminants, hydrogen production, and chemical synthesis, due to it has reliable chemical inertness, excellent thermal stability, proper band-gap and special visible absorption capacity[9-11]. However, the photocatalytic performance of $\mathrm{g}-\mathrm{CN}$ are restricted by the fast recombination of photo-generated carriers and the poor light absorption capacity[12-14]. Hence, many methods including morphology control, the preparation of g-CN based heterojunction, deposition of noble metal and modified with carbon materials have been applied to enhance the photocatalytic performance of g$\mathrm{CN}$ [15-17]. Carbon materials, as an earth-abundant resource, have been extensively explored to modified with g-CN. Among the many carbon materials, graphene quantum dots (GQDs) are a novel 0D fluorescent carbon nanomaterials that have $<10 \mathrm{~nm}$ lateral size and few-layer structure[18-20]. In the process of photocatalysis, GQDs can serve as an emulsion and an electron accepter to expand the range of visible absorption and reduce the recombination of photo-generated charges[21-23].

In this work, GQDs were prepared from 1,3,6trinitropyrene by hydrothermal treatment, and the samples g-CN/GQDs-X was prepared through a one-pot cosintering the mixture of GQDs and melamine. After modified with GQDs, the g-CN show more intensive visible light absorption and better transfer ability of photo-generated electrons. The prepared materials were characterized, and antibiotics TC was served as a model contaminant to test the photocatalytic ability. Meanwhile, the active species of TC degradation process were evaluated and a possible photocatalytic degradation mechanism were proposed.

\section{Experimental}

\subsection{Synthesis of the catalysts}

The GQDs were prepared from 1,3,6-trinitropyrene by hydrothermal treatment in accordance with previous reports[24]. The g-CN/GQDs samples was obtained by calcined a mixture of melamine and GQDs. Typically, $3 \mathrm{~g}$ melamine was added to $50 \mathrm{~mL}$ of DI water. Then, a certain amount of GQDs solution $(10 \mathrm{mg} / \mathrm{mL})$ was dropped into the melamine suspension, and vigorously stirred $2 \mathrm{~h}$, then vacuum dried at $80^{\circ} \mathrm{C}$ to dewater. After that, the mixture was transferred to a covered ceramic crucible, calcined at $520^{\circ} \mathrm{C}$ in a muffle furnace for $2 \mathrm{~h}$ with a heat-up speed of $5^{\circ} \mathrm{C} \mathrm{min}^{-1}$. The resultant samples were collected and

\footnotetext{
${ }^{*}$ Corresponding author: jxsu@sdu.edu.cn
} 
milled into a fine powder. The final catalysts were named as $\mathrm{g}-\mathrm{CN} / \mathrm{GQDs}-\mathrm{X}$, where $\mathrm{X}$ represents the volume of GQDs solution added. The pristine $\mathrm{g}-\mathrm{CN}$ was prepared by the same process without adding GQDs.

\subsection{Characterization}

Powder X-ray diffraction (XRD) patterns of as-prepared powders were analysed by Ultima IV X-Ray. The functional groups of prepared materials were collected through Fourier transform infrared spectrophotometer (FT-IR, Perkin-Elmer Spectrum BX00 spectrometer). The spectrophotometer (Shimadzu UV-2550) equipped with an integrating sphere accessory was used to obtain the diffuse reflectance spectra of the samples using BaSO4 as a reference. Photoluminescence (PL) spectra of the samples were collected by using Edinburgh FL/FS900 spectrophotometer. Photocurrent response experiments were recorded at $\mathrm{CHI} 660 \mathrm{E}$ electrochemical workstation. Pt plate and $\mathrm{Ag} / \mathrm{AgCl}$ electrode were used as counter electrode and reference electrode, respectively. And the synthesized samples dispersed on $1.5 \mathrm{~cm} \times 1.5 \mathrm{~cm}$ Indium tin oxide (ITO) glass were used as working electrode.

\subsection{Photodegradation experiment}

The photodegradation activity of the synthetic materials were study by monitoring the degradation of a TC solution upon irradiation with visible light $(>400 \mathrm{~nm})$. The light source is a 300W Xenon lamp (CEL-HXF300) equipped with a $400 \mathrm{~nm}$ cut off filter. Briefly, 50mg samples were added into the reaction breaker including $100 \mathrm{ml} \mathrm{TC}$ solution $(10 \mathrm{mg} / \mathrm{L})$. Before the photocatalytic, the samples were stirred in the dark for $30 \mathrm{~min}$ to ensure the adsorption-desorption equilibrium. The reaction TC suspension $(3 \mathrm{~mL})$ was taken out every $10 \mathrm{~min}$ and filtered using a $0.45 \mu \mathrm{m}$ membrane filter, then analysed by a Shimadzu UV-2550 spectrophotometer to monitor the concentration of TC. After use, the samples were collected and washed several times with ultrapure water and ethanol for next experiment, and Four successive cycles of tetracycline degradation experiments were carried out.

\section{Results and discussion}

X-ray diffraction (XRD) patterns of the prepared materials were exhibited in Fig.1. GQDs show a clearly (002) signal at $26.4^{\circ}$ corresponding to the typical XRD pattern of the pristine graphite[24]. In addition, it can be found that there are two peaks at $2 \theta=13.4^{\circ}$ and $27.4^{\circ}$ displayed in g-CN and g-CN/GQDs-X. The peak at $13.4^{\circ}$ could be assigned to (100) diffraction planes of $\mathrm{g}-\mathrm{CN}$, attributed to the interplanar structural packing[25]. The peak at $27.4^{\circ}$ corresponds to the (002) diffraction plane of $\mathrm{g}-\mathrm{CN}$, due to the stacking of the conjugated aromatic system[26]. However, no characteristic peak of GQDs was found in the $\mathrm{g}-\mathrm{CN} / \mathrm{GQDs}-\mathrm{X}$ composite materials, which may due to the low GQDs content[27].

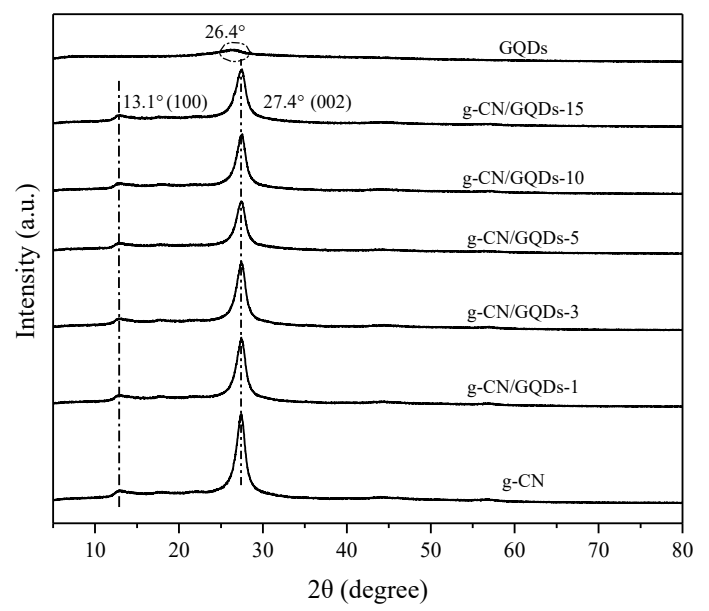

Figure 1. XRD of the prepared samples.

Fig.2 presents the FT-IR spectra of the synthetic materials. The GQDs contains abundant surface groups, among which the peak at $3400 \mathrm{~cm}^{-1}$ corresponds to the stretching vibration of $-\mathrm{OH}$ group, and there is also vibration peak of $\mathrm{C}=\mathrm{C}, \mathrm{C}-\mathrm{H}$ and $\mathrm{C}-\mathrm{OH}$. All the gCN/GQDs-X samples showed some characteristic peaks at around $807 \mathrm{~cm}^{-1}, 1200 \sim 1600 \mathrm{~cm}^{-1}$ and $3210 \mathrm{~cm}^{-1}$, respectively. The peak at $807 \mathrm{~cm}^{-1}$ reflected the breathing mode of the tri-s-triazine ring system, the peaks at $1200 \sim 1600 \mathrm{~cm}^{-1}$ was assigned to the characteristic stretching vibration modes of $\mathrm{CN}$ and $\mathrm{C}-\mathrm{N}$ heterocycles, and the peak at $3210 \mathrm{~cm}^{-1}$ corresponds to the terminal N$\mathrm{H}_{2}$ or N-H stretching vibration[28-30]. Whereas, g-CN and g-CN/GQDs-X showed same FT-IR spectra, indicating that the addition of the GQDs has no influence on the functional groups of g-CN[27, 28].

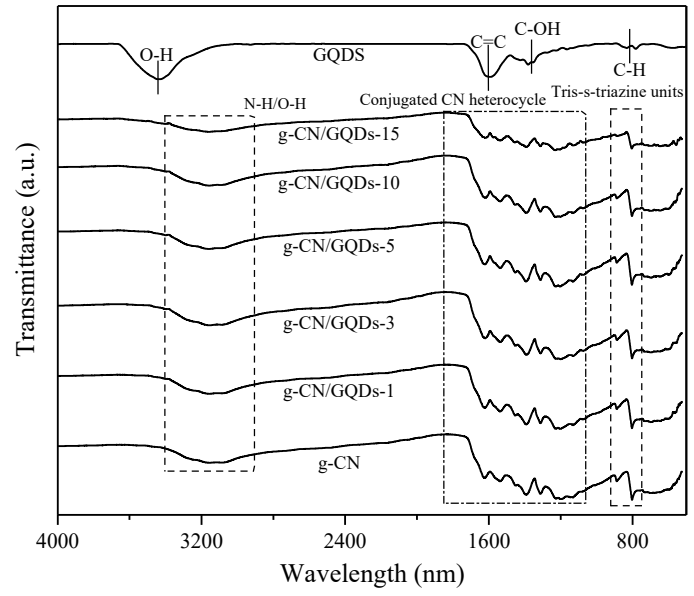

Figure 2. FT-IR of the prepared samples.

The morphologies of as-synthesized photocatalysts were characterized via TEM analyses. As show in Fig.3(a, b), the GQDs are dispersed uniformly and with a lateral size about $2 \sim 5 \mathrm{~nm}$. It can be discovered that the obvious crystalline lattices of GQDs in the high-resolution TEM picture, suggesting the GQDs has high crystallinity[24]. Fig.3(c) indicated that the g-CN was composed of stacked nanosized sheets. Compared with g-CN, the g-CN/GQDs5 shows more broken and smaller lamellas (Fig.3(b)). Interestingly, no specific GQDs appearance was found in the electron microscope image of g-CN/GQDs-5, which 
may due to the low addition of GQDs and uniform distribution in the g-CN/GQDs-5[17, 25, 31].

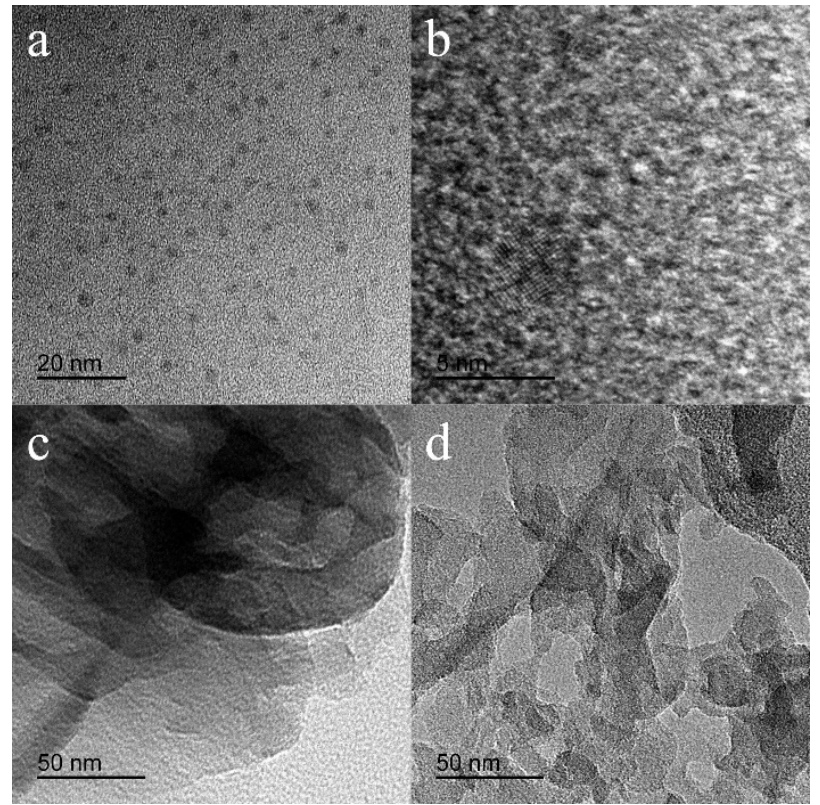

Figure 3. TEM images of (a, b) GQDs, (c) g-CN and (d) gCN/GQDs-5.

The optical absorption performance of prepared samples was collected through UV-Vis diffuse reflectance spectroscopy. It can be seen from Fig.4, both g-CN and g-CN/GQDs-X samples present excellent absorption at the wavelength range of $200 \sim 450 \mathrm{~nm}$. It is worth mentioning that the absorption property changes of g-CN/GQDs-X shows a slightly red shift. Besides, all the g-CN/GQDs-X samples have higher optical absorption in the full wavelength compared with g-CN, suggesting that the prepared g-CN/GQDs-X exhibit a better visible light utilization performance than that of $\mathrm{g}-\mathrm{CN}$, which may more conducive to photocatalysis process.

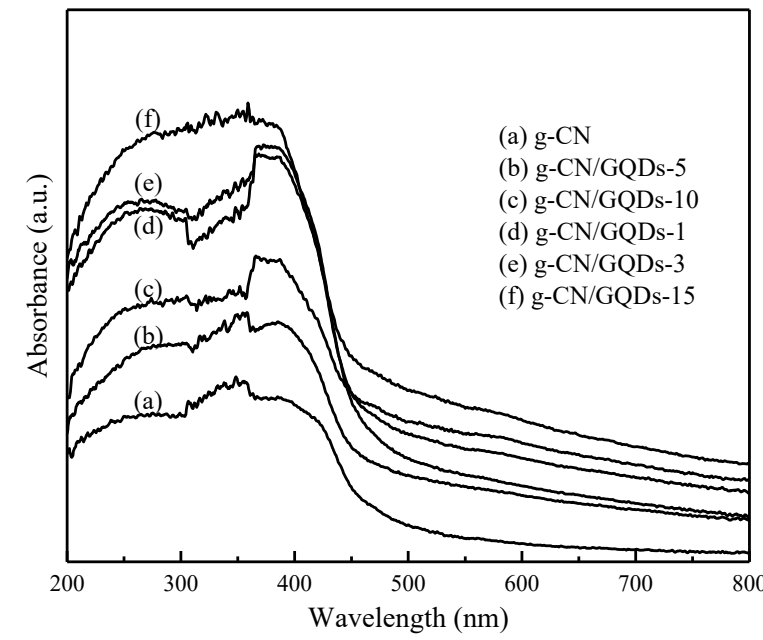

Figure 4. UV-Vis absorption spectra of the prepared samples.

Fig.5(a) shows the PL spectra of g-CN and gCN/GQDs-5 samples at $340 \mathrm{~nm}$ excitation wavelength. The main peak of g-CN and g-CN/GQDs-5 is at $\sim 466 \mathrm{~nm}$ and the g-CN/GQDs-5 sample show much low PL intensity than $\mathrm{g}-\mathrm{CN}$, indicating that the latter possess the lower recombination rate of the charges. As show in Fig.5(b), the photocurrent density of g-CN/GQDs-5 was evidently higher than that of $\mathrm{g}-\mathrm{CN}$ indicating the better photo-generated carrier separation ability.
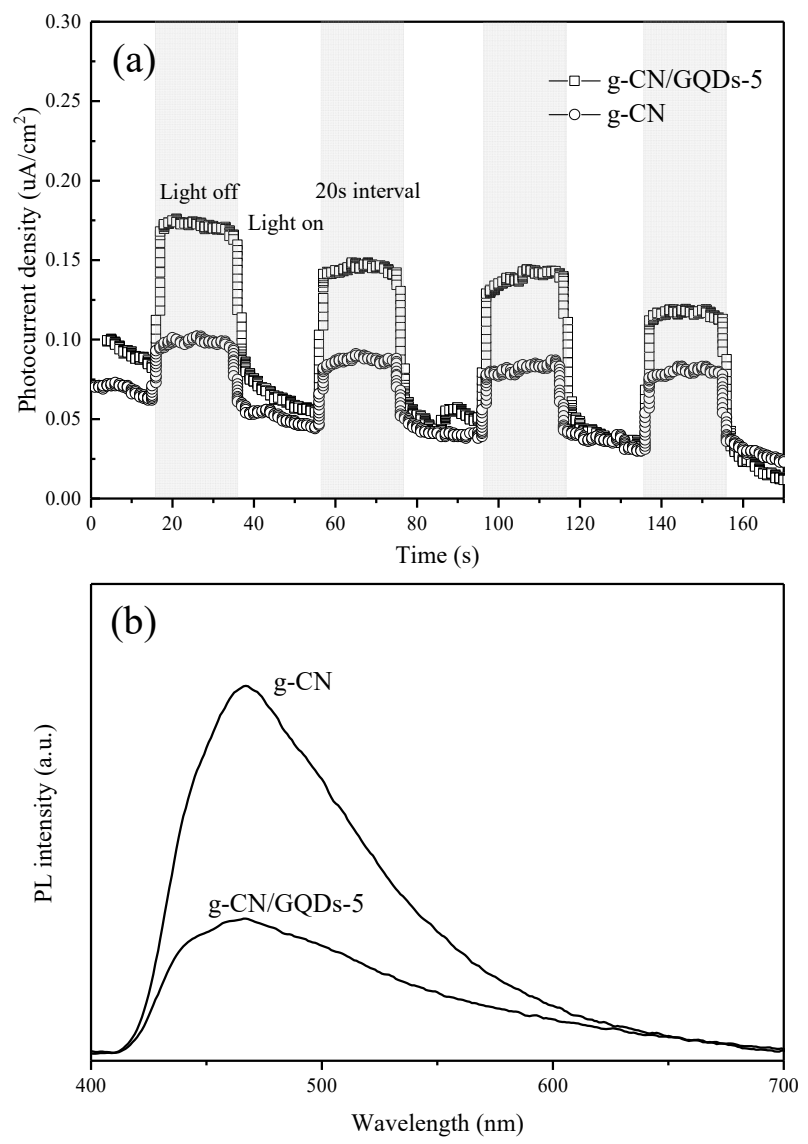

Figure 5. (a) The photocurrent response and (b) PL spectra of $\mathrm{g}-\mathrm{CN}$ and $\mathrm{g}-\mathrm{CN} / \mathrm{GQDs}-5$.

To investigate the photocatalysis performance of the prepared samples, the tetracycline (TC), a typical antibiotic, was choose as the targeted contaminant. It can be observed that all the samples were absorbed very little antibiotic under dark condition. In addition, there was almost no decomposition of TC under visible light irradiation without adding photocatalysts, suggesting the refractory nature of TC under visible light irradiation. After adding the as-prepared samples, the degradation rate of TC was significantly enhanced. As show in Fig.6(a), all the catalysts with GQDs modified show much better degradation rate of TC than g-CN (51\%), which may be attributed to the introduction of GQDs into g-CN enhanced the visible light utilization performance and improved the separation of $\mathrm{h}^{+}$and $\mathrm{e}^{-}$. As expected, the TC degradation rate of the optimal catalyst g-CN/GQDs-5 reached $78 \%$, which had a nearly $30 \%$ improvement than that of g-CN. The pseudo-first-order models was used to study the kinetics of the photocatalytic TC degradation by prepared samples, the formula as follow:

$$
\ln \frac{C_{0}}{C}=k t
$$

where $\mathrm{t}$ represents the reaction time, $\mathrm{C}_{0}$ and $\mathrm{C}$ represent the $\mathrm{TC}$ concentrations at $0 \mathrm{~min}$ and $\mathrm{t} \mathrm{min}, \mathrm{k}$ represents the reaction rate constant of pseudo-first-order kinetics. As 
show in Fig.6(b), the photocatalytic degradation curves of both $\mathrm{g}-\mathrm{CN}$ and $\mathrm{g}-\mathrm{CN} / \mathrm{GQDs}-\mathrm{X}$ follow pseudo-first-order kinetics $\left(\mathrm{R}^{2}>0.98\right)$, and the highest kinetic constants of $\mathrm{g}$ CN/GQDs-5 were estimated to be $0.02427 \mathrm{~min}^{-1}$, which were 1.8 times higher than that of $\mathrm{g}-\mathrm{CN}\left(0.01644 \mathrm{~min}^{-1}\right)$.
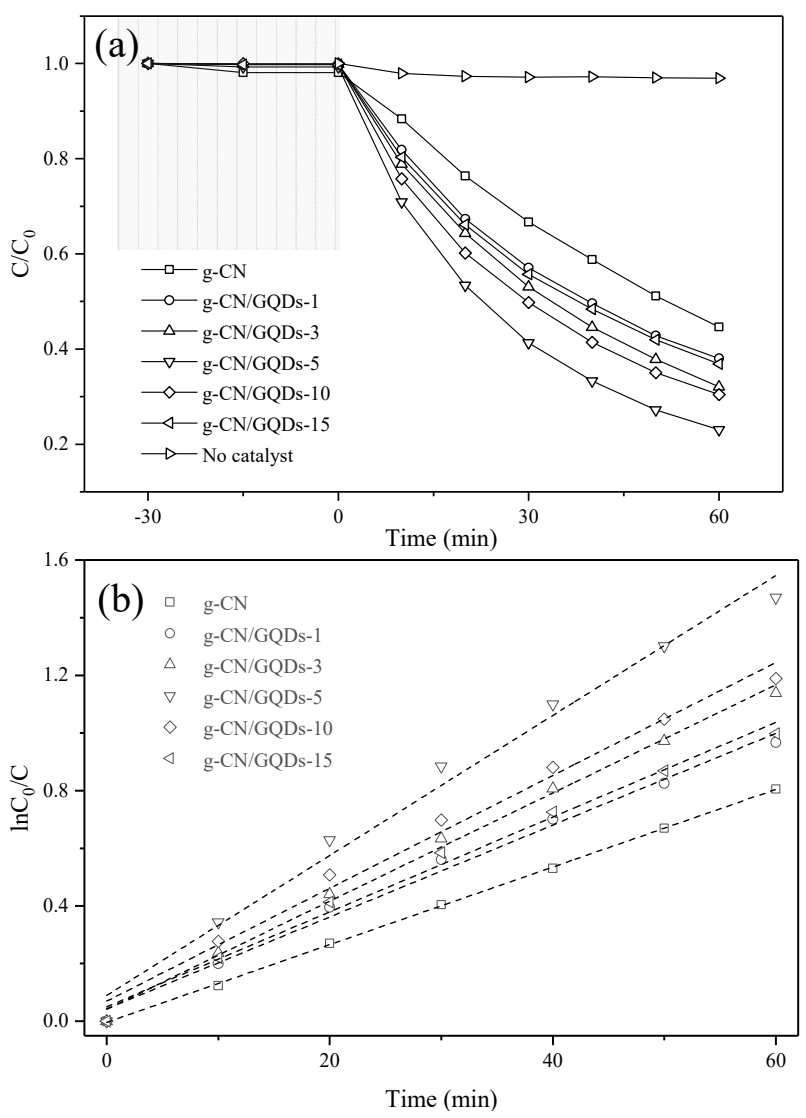

Figure 6. (a) Photocatalytic degradation performance and (b) pseudo-first-order kinetic fitting curves.

In the practical applications of photocatalyst, reusable performance is also crucially important. The gCN/GQDs-5 composites of the TC degradation were reused four times under the same reaction conditions. As illustrated in Fig.7(a), the photocatalytic activity of gCN/GQDs-5 does not show any significant decrease after four cycles, indicating that it has excellent stability and recyclability. To better understanding the impact of active species in the photo-degradation of TC, the scavengers EDTA, benzoquinone (BQ) or isopropanol (IPA) was respectively injected to the $\mathrm{g}-\mathrm{CN} / \mathrm{GQDs}-5$ reaction system to trap the $\mathrm{h}^{+}, \cdot \mathrm{O}^{2-}$ and $\cdot \mathrm{OH}$. From Fig.7(b), the degrading efficiency of g-CN/GQDs-5 was drastically reduced after adding $\mathrm{BQ}$, suggesting the major impact of $\cdot \mathrm{O}^{2-}$ in the photo-degradation of TC in g-CN/GQDs-5 system. It is worthy to point out that, IPA and EDTA shows little effect on the TC degradation, suggesting that $\cdot \mathrm{OH}$ and $\mathrm{h}^{+}$radicals also contribute to the degradation of TC.
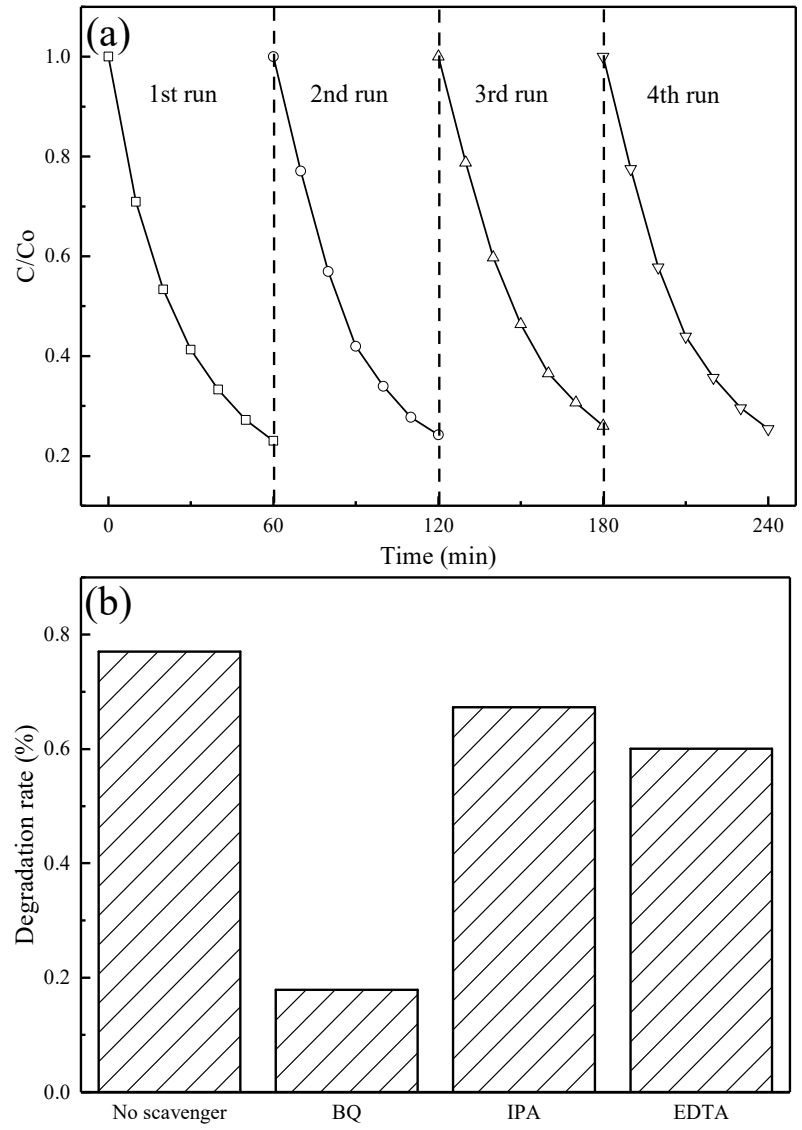

Figure 7. (a) Recycling performance and (b) activity capture experiment of g-CN/GQDs-5 sample.

Based on the experimental results, a possible mechanism of g-CN/GQDs-5 system is possessed. When the g-CN were exposed to visible light, electrons get excited and move to the conduction band, but a relatively large part of photo-generated electrons have been consumed on account of the rapid recombination of photo-generated $\mathrm{e}^{-}$and $\mathrm{h}^{+}$pair. However, GQDs can act as an emulsion and an electron accepter to enhance light absorption capacity and reduce the photo-generated electron-hole pair recombination in the g-CN/GQDs-5 system, as evidenced by UV-Vis absorption spectra and PL spectra. In the photocatalytic process of g-CN/GQDs5 , the electrons of g-CN/GQDs-5 composite was excited from valence band to conduction band after exposed to visible light, and subsequently transfer to the tightly coupled GQDs rapidly. The electrons would react with the dissolved $\mathrm{O}_{2}$ to generate $\cdot \mathrm{O}^{2-}$ radicals, which played a major impact in the degradation of TC. Meanwhile, the $\cdot \mathrm{O}^{2-}$ could reacted with $\mathrm{H}_{2} \mathrm{O}$ to form $\mathrm{H}_{2} \mathrm{O}_{2}$ and further conversion to hydroxyl radicals to participated in the TC degradation process. Besides, the $\mathrm{h}^{+}$produced of $\mathrm{g}$ CN/GQDs-5 also made contribution to decomposing TC into small molecules.

\section{Conclusion}

In this work, GQDs were prepared hydrothermal reaction of 1,3,6-trinitropyrene and g-CN/GQDs-X composites were prepared by calcined a mixture of melamine and GQDs. The prepared g-CN/GQDs-X show enhanced 
photo-degradation tetracycline activity as compared with the g-CN. The enhanced photocatalytic performance of g$\mathrm{CN} / \mathrm{GQD}$ is mainly related to the rapid transfer and separation of photo-generated carriers, and the enhance of optical absorption capacity. This work provides a new way for the preparing of high efficiency carbon nitridebased photocatalyst by modified with quantum dots and for its application in environmental treatment.

\section{References}

1. Q. Q. Zhang, G. G. Ying, C. G. Pan, Y. S. Liu and J. L. Zhao, Environ Sci Technol 49 (11), 6772-6782 (2015).

2. S. Li and J. Y. Hu, J Hazard Mater 318, 134-144 (2016).

3. S. Li, W. Z. Shi, W. Liu, H. M. Li, W. Zhang, J. R. Hu, Y. C. Ke, W. L. Sun and J. R. Ni, Sci Total Environ 615, 906-917 (2018).

4. C. Reyes, J. Fernandez, J. Freer, M. A. Mondaca, C. Zaror, S. Malato and H. D. Mansilla, J Photoch Photobio A 184 (1-2), 141-146 (2006).

5. M. Ahmadi, H. R. Motlagh, N. Jaafarzadeh, A. Mostoufi, R. Saeedi, G. Barzegar and S. Jorfi, J Environ Manage 186, 55-63 (2017).

6. S. Zhang, P. C. Gu, R. Ma, C. T. Luo, T. Wen, G. X. Zhao, W. C. Cheng and X. K. Wang, Catal Today 335, 65-77 (2019).

7. Y. Zhang, M. J. Zhang, L. Tang, J. J. Wang, Y. Zhu, C. Y. Feng, Y. C. Deng, W. Z. He and Y. Hu, Chem Eng J 391 (2020).

8. P. P. Tun, J. T. Wang, T. T. Khaing, X. Y. Wu and G. K. Zhang, J Alloy Compd 818 (2020).

9. T. B. Nguyen, C. P. Huang, R. A. Doong, C. W. Chen and C. D. Dong, Chem Eng J 384 (2020).

10. M. Bellardita, E. I. Garcia-Lopez, G. Marci, I. Krivtsov, J. R. Garcia and L. Palmisano, Appl Catal B-Environ 220, 222-233 (2018).

11. Z. H. Li, D. L. Huang, C. Y. Zhou, W. J. Xue, L. Lei, R. Deng, Y. Yang, S. Chen, W. J. Wang and Z. W. Wang, Chem Eng J 382 (2020).

12. D. L. Huang, Z. H. Li, G. M. Zeng, C. Y. Zhou, W. J. Xue, X. M. Gong, X. L. Yan, S. Chen, W. J. Wang and M. Cheng, Appl Catal B-Environ 240, 153-173 (2019).

13. J. F. Zhang, J. W. Fu, Z. L. Wang, B. Cheng, K. Dai and W. K. Ho, J Alloy Compd 766, 841-850 (2018).

14. D. H. He, C. Zhang, G. M. Zeng, Y. Yang, D. L. Huang, L. L. Wang and H. Wang, Appl Catal BEnviron 258 (2019).

15. P. Raizada, A. Sudhaik, P. Singh, P. Shandilya, V. K. Gupta, A. Hosseini-Bandegharaei and S. Agrawal, J Photoch Photobio A 374, 22-35 (2019).

16. F. T. Yi, H. H. Gan, H. F. Jin, W. Y. Zhao, K. F. Zhang, H. X. Jin, H. N. Zhang, Y. X. Qian and J. Q. Ma, Sep Purif Technol 233 (2020).
17. B. T. Huang, J. B. He, S. Y. Bian, C. J. Zhou, Z. Y. Li, F. N. Xi, J. Y. Liu and X. P. Dong, Chinese Chem Lett 29 (11), 1698-1701 (2018).

18. X. T. Zheng, A. Ananthanarayanan, K. Q. Luo and P. Chen, Small 11 (14), 1620-1636 (2015).

19. S. Fang, Y. Xia, K. L. Lv, Q. Li, J. Sun and M. Li, Appl Catal B-Environ 185, 225-232 (2016).

20. A. Ananthanarayanan, X. W. Wang, P. Routh, B. Sana, S. Lim, D. H. Kim, K. H. Lim, J. Li and P. Chen, Adv Funct Mater 24 (20), 3021-3026 (2014).

21. S. Kim, Y. J. Song and M. J. Heller, Adv Mater 29 (31) (2017).

22. L. Ai, R. Shi, J. Yang, K. Zhang, T. R. Zhang and S. Y. Lu, Small 2007523 (2021).

23. B. Li, W. Peng, J. Zhang, J. C. Lian, T. Huang, N. Cheng, Z. Y. Luo, W. Q. Huang, W. Y. Hu, A. L. Pan, L. Jiang and G. F. Huang, Adv Funct Mater 2100816 (2021).

24. L. Wang, Y. L. Wang, T. Xu, H. B. Liao, C. J. Yao, Y. Liu, Z. Li, Z. W. Chen, D. Y. Pan, L. T. Sun and M. H. Wu, Nat Commun 5 (2014).

25. A. L. Yuan, H. Lei, F. N. Xi, J. Y. Liu, L. S. Qin, Z. Chen and X. P. Dong, J Colloid Interf Sci 548, 56-65 (2019).

26. C. Y. Zhou, P. Xu, C. Lai, C. Zhang, G. M. Zeng, D. L. Huang, M. Cheng, L. Hu, W. P. Xiong, X. F. Wen, L. Qin, J. L. Yuan and W. J. Wang, Chem Eng J 359, 186-196 (2019).

27. W. Liu, Y. Y. Li, F. Y. Liu, W. Jiang, D. D. Zhang and J. L. Liang, Water Res 151, 8-19 (2019).

28. C. Zhao, Z. Z. Liao, W. Liu, F. Y. Liu, J. Y. Ye, J. L. Liang and Y. Y. Li, J Hazard Mater 381 (2020).

29. F. Wei, J. D. Li, C. C. Dong, Y. J. Bi and X. J. Han, Chemosphere 242 (2020).

30. S. Z. Liu, H. Q. Sun, K. O'Donnell, H. M. Ang, M. O. Tade and S. B. Wang, J Colloid Interf Sci 464, 10-17 (2016).

31. H. Zhang, L. X. Zhao, F. L. Geng, L. H. Guo, B. Wan and Y. Yang, Appl Catal B-Environ 180, 656-662 (2016). 